\title{
Mechanism and regioselectivity of 1,3-dipolar cycloaddition reactions of sulphur-centred dipoles with furan- 2,3-dione: A theoretical study using DFT
}

\author{
SAEED REZA EMAMIAN*, SAFA ALI-ASGARI and EHSAN ZAHEDI \\ Chemistry Department, Shahrood Branch, Islamic Azad University, Shahrood 36199-43189, Iran \\ e-mail: s_emamian@iau-shahrood.ac.ir
}

MS received 21 July 2013; revised 8 September 2013; accepted 17 September 2013

\begin{abstract}
The mechanism and regioselectivity of 1,3-dipolar cycloaddition reactions of sulphur-centred 1,3dipoles including thiocarbonyl S-imide (D1), thiocarbonyl S-oxide (D2) and thiocarbonyl S-sulphide (D3) with an electron-deficient dipolarophile, furan-2,3-dione (DPh), were studied in the light of some theoretical approaches, namely, activation energy, density functional theory (DFT) reactivity indices and Houk's rule based on the frontier molecular orbital (FMO) theory at the B3LYP/6-311++ $\mathrm{G}^{* *}$ level. The present analysis reveals that the cycloaddition reactions under study can be classified in the normal electron demand category. An excellent agreement was observed between the kinetic results and the electronic approaches; in fact, maximum hardness principle (MHP), Chattaraj's polar model, Houk's rule and the Gazquez-Mendez rule confirm the resultant regioselectivity based on the calculated activation energies.
\end{abstract}

Keywords. 1,3-Dipolar cycloaddition; sulphur-centred 1,3-dipoles; regioselectivity; DFT reactivity indices; FMO theory.

\section{Introduction}

Five-membered heterocyclic compounds can be generated by addition of a 1,3-dipole to a dipolarophile under a 1,3-dipolar cycloaddition (1,3-DC) reaction which is well known as pericyclic reaction. A 1,3-dipole is an arrangement of three atoms over which four $\pi$ electrons are distributed while dipolarophiles are systems including unsaturated bond(s). ${ }^{1,2}$ 1,3-DC reactions are synthetically useful in organic synthetic field because of their high stereospecificity, stereoselectivity and regioselectivity. ${ }^{3}$ For the first time in 1960, Huisgen and co-workers introduced the concept of 1,3-DC reactions. ${ }^{4,5}$ From the mechanistic point of view, two types of these reactions have been introduced: stepwise and concerted. ${ }^{6}$ According to the number of researches on stereochemistry and kinetics, it can be concluded that the 1,3-dipolar cycloaddition reactions proceed via concerted mechanism. ${ }^{3,7,8}$ The concerted mechanism was primarily suggested by Huisgen ${ }^{9,10}$ and it has been specified with the following characterizations: singlestep, five-centre cycloaddition, and involves $4 \pi$ electrons from the 1,3-dipole and $2 \pi$ electrons from the dipolarophile. In this mechanism, in agreement with the Woodward-Hoffmann rules, ${ }^{11}$ combination of three

*For correspondence $\mathrm{p}_{\mathrm{z}}$ orbitals of 1,3-dipole (containing $4 \pi$ electrons) and two $p_{z}$ orbitals of the dipolarophile (containing $2 \pi$ electrons) takes place suprafacially and this is the reason why these reactions are symbolized as $\left[{ }_{\pi} 4_{\mathrm{S}}+{ }_{\pi} 2_{\mathrm{S}}\right]$. Based on Fleming's explanations, ${ }^{12}$ regioselectivity and reactivity in concerted 1,3-DC reactions can be interpreted by using frontier molecular orbital (FMO) theory. In the light of FMO theory, Sustmann classified the cycloaddition reactions in three various types. Type $\mathrm{I}$ is the FMO interaction between the highest occupied molecular orbital of the 1,3-dipole $\left(\mathrm{HOMO}_{\text {dipole }}\right)$ and the lowest unoccupied molecular orbital of the dipolarophile $\left(\mathrm{LUMO}_{\text {dipolarophile }}\right)$ which is named as the normal-electron demand (NED) 1,3-DC reactions and a large number of 1,3-DC reactions are classified in this type. Type II is known by the interaction between the LUMO of the 1,3-dipole and the HOMO of the dipolarophile. This type is named as the inverse-electron demand (IED) 1,3-DC reactions. Finally, type III is characterized by the similar differences of the HOMO and LUMO energies of the 1,3-dipole/dipolarophile pair. ${ }^{13-15}$ In this case, both $\mathrm{LUMO}_{\text {dipolarophile }}-\mathrm{HOMO}_{\text {dipole }}$ and $\mathrm{LUMO}_{\text {dipole }}-\mathrm{HOMO}_{\text {dipolarophile }}$ interactions must be considered in determining the reactivity and regiochemistry of the process and consequently either NED or IED can be occurred. ${ }^{5}$ These three types of interactions are depicted in scheme 1 . 

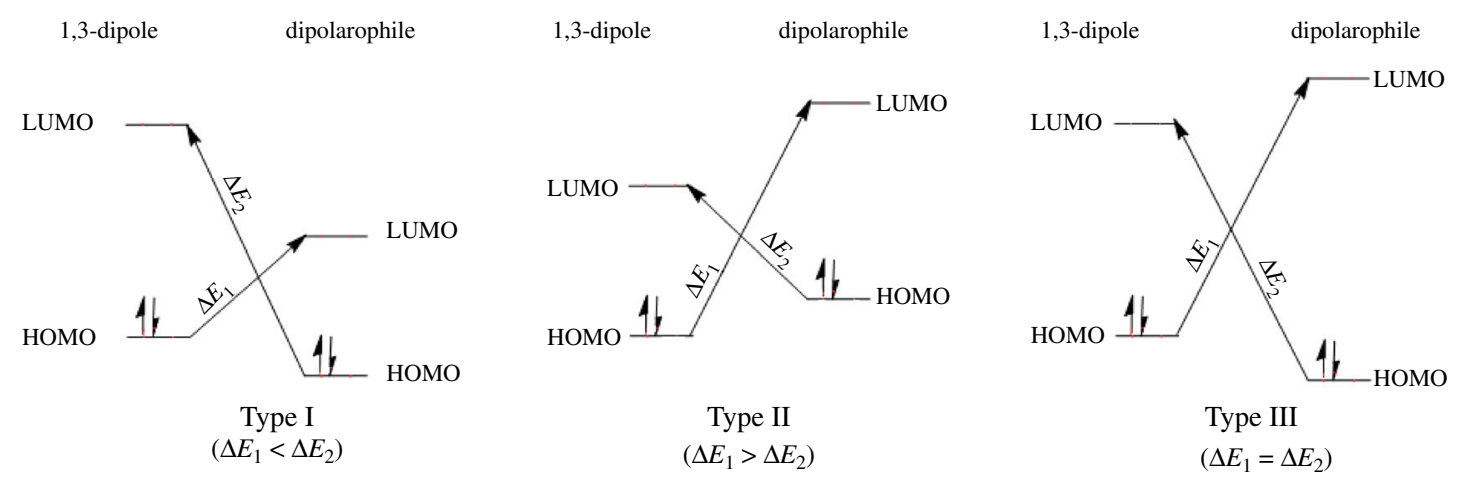

Scheme 1. Three types of interactions between HOMO and LUMO orbitals of a 1,3-dipole/dipolarophile pair.

In addition to FMO theory, many researchers use the HSAB (hard and soft acids and bases) principle which has been formulated by density functional theory (DFT) $)^{10,15}$ to predict reactivity and regioselectivity in the 1,3-DC reactions. Moreover, based on the DFT, a number of global and local reactivity descriptors such as global electrophilicity, global hardness, global softness, local hardness, local softness, condensed Fukui functions, philicity indexes, etc., have been developed to foresee the regioselectivity in 1,3-DC reactions and these can be found in the literature. ${ }^{16-20}$ Another interesting and helpful tool to predict reactivity and stability of chemical species is the maximum hardness principle (MHP) ${ }^{21}$ which has been formulated by Pearson in $1987 .{ }^{22}$ This principle states that "there seems to be a rule of nature that molecules arrange themselves to be as hard as possible". ${ }^{22,23}$ Applicability of MHP has been confirmed to explore the chemical reactivity and direction to which a chemical reaction evolves. ${ }^{21,24}$ An interesting idea within the MHP is the activation hardness, introduced by Zhou and Parr, ${ }^{22,25}$ which is defined as the difference between the hardness of reactants and corresponding transition state: $\Delta \eta^{\neq}=$ $\eta_{R}-\eta_{T S}$. Kinetically favoured regioisomer must be obtained from a more stable transition state which is also higher in hardness. Indeed, this is the application of MHP to predict the regioselectivity in a cycloaddition reaction and has been successfully used to study these reactions. ${ }^{16,26-28}$

Sulphur-centred 1,3-dipoles are uncommon species in comparison with other 1,3-dipoles, which have been extensively explored in organic synthesis. The 1,3-DC reaction of an unsaturated bond with a sulphurcentred 1,3-dipole is a useful method for the synthesis of sulphur-containing five-membered heterocyclic compound. ${ }^{29}$ The five-membered cyclic compounds containing two directly bonded heteroatoms, including sulphur-nitrogen, sulphur-oxygen, and sulphursulphur are called isothiazolidine, 1,2-oxathiolane, and 1,2-dithiolane, respectively.
In the present study, regioselectivity and mechanism of 1,3-DC reactions of sulphur-centred 1,3-dipoles including thiocarbonyl S-imide (D1), thiocarbonyl Soxide (D2) and thiocarbonyl S-sulphide (D3) with furan-2,3-dione $(\mathrm{DPh})$ are studied in the light of several theoretical approaches such as activation energy Houk's rule based on the FMO theory ${ }^{30}$ and the DFT reactivity indices.

\section{Theoretical background}

DFT can be used as a powerful tool to understand molecular structure, molecular properties, and molecular reactivity. ${ }^{31}$ Based on the conceptual DFT, global descriptors such as electronic chemical potential $(\mu)$, hardness $(\eta)$, and softness $(S)$ are defined as follows: ${ }^{32}$

$$
\begin{gathered}
\mu=(\partial E / \partial N)_{\nu} \\
2 \eta=\frac{1}{S}=\left(\partial^{2} E / \partial N^{2}\right)_{\nu}=(\partial \mu / \partial N)_{\nu},
\end{gathered}
$$

where $E$ and $v$ are total electronic energy of a system having N-electron and external potential, respectively. By using Koopman's theorem, ${ }^{33}$ these equations can be rewritten as:

$$
\begin{gathered}
\mu=\frac{1}{2}\left(\varepsilon_{\text {НОмО }}+\varepsilon_{\text {LUMO }}\right) \\
\eta=\frac{1}{2 S}=\frac{1}{2}\left(\varepsilon_{\text {LUMO }}-\varepsilon_{\text {НОмО }}\right),
\end{gathered}
$$

where $\varepsilon_{\text {HOMO }}$ and $\varepsilon_{\text {LUMO }}$ are, respectively, the highest occupied and the lowest unoccupied molecular orbital energies. The following expression, introduced by Parr et al., ${ }^{34}$ provides another important and interesting global descriptor, which is called electrophilicity index:

$$
\omega=\frac{\mu^{2}}{2 \eta} \text {. }
$$


Electrophilicity index is a measure of the electrophilic power of a system ${ }^{35}$ and can be described as the maximal ability of a molecule to accept electrons in the neighbourhood of an electron reservoir. ${ }^{21}$ Equation (5) clearly implies that a molecule can act as a better electrophile with a more negative value of $\mu$ (larger value of $\mu^{2}$ ) and a smaller value of $\eta$, which reflects the higher tendency to acquire charge from the environment and the lower resistance in electronic charge exchange with the environment, respectively.

Local descriptors must be considered in order to know which site in a molecule, in a considered reaction, is more reactive than others. Local descriptors such as Fukui functions $f(r)$, local softness $s(r)$ and local philicity indices define the reactivity and selectivity of a specific site in a molecule. Fukui function is defined as the first derivative of the electronic density $\rho(r)$ of a system with respect to the number of electrons $N$ at a constant external potential $v(r):{ }^{36}$

$$
f(r)=\left[\frac{\partial \rho(r)}{\partial N}\right]_{v(r)} .
$$

The condensed forms of Fukui functions of an atom, say $k$, in a molecule with $N$-electron were proposed by Yang and Mortier regarding the type of electron transfer: ${ }^{37}$

$$
f_{k}^{+}=\left[\rho_{k}(N+1)-\rho_{k}(N)\right] \quad \text { for nucleophilic attack }
$$

$f_{k}^{-}=\left[\rho_{k}(N)-\rho_{k}(N-1)\right] \quad$ for electrophilic attack,

where $\rho_{k}(N), \rho_{k}(N-1), \rho_{k}(N+1)$ are the gross electronic population of the site $k$ in neutral, cationic and anionic systems, respectively.

The local softness, $s_{k}^{\alpha}$, and local philicity index, $\omega_{k}^{\alpha}$, are easily obtained by multiplying global quantities and condensed Fukui functions in a molecule. These local indexes, $s_{k}^{\alpha}$ and $\omega_{k}^{\alpha}$, can readily be calculated as follows: ${ }^{38}$

$$
\begin{gathered}
s_{k}^{\alpha}=S f_{k}^{\alpha} \\
\omega_{k}^{\alpha}=\omega f_{k}^{\alpha},
\end{gathered}
$$

where $\alpha=+$ and $\alpha=-$ refer to the nucleophilic and electrophilic attacks, respectively.

\section{Computational details}

In this study, geometries of all reactants (D1-D3 and $\mathrm{Dph}$ ) and transition state structures (TSs) were fully optimized by using DFT with the B3LYP ${ }^{39}$ exchange correlation functional and $6-311++\mathrm{G}^{* *}$ basis set. To confirm the nature of stationary species and evaluate the activation energy barriers, frequency calculations were carried out at $298.15 \mathrm{~K}$ and $1.0 \mathrm{~atm}$. For minimum state structures and for the TSs, only real frequency values and only a single imaginary frequency value were accepted, respectively. Normal modes corresponding to the imaginary frequencies in the transition state structures are related to the vibrations of new developing bonds. Stabilities of restricted wave functions towards unrestricted alternatives for reactants were verified using STABLE keyword ${ }^{40}$ and instability was not found. Owing to the poor estimation of Kohn-Sham orbitals for FMO energy values, ${ }^{41}$ HOMO and LUMO energies and corresponding global descriptors described in equations 3-5 were calculated by using $\mathrm{HF} / 6-311++\mathrm{G}^{* *}$ single point calculation on the B3LYP $/ 6-311++\mathrm{G}^{* *}$ optimized geometries. In calculations of the condensed Fukui functions, the cationic and anionic systems were kept at the optimized geometries of the corresponding neutral systems. Electronic populations were analysed by the natural population analysis (NPA), ${ }^{42}$ Hirshfeld charges ${ }^{43}$ and also by the charges derived from the electrostatic potential and calculated according to Merz-Kollman (MK option). ${ }^{44}$ To verify regioselectivity of the 1,3-DC reactions considered in this study based on Houk's rule, coefficients of the frontier molecular orbitals (HOMO and LUMO) were calculated at the HF/STO-3G level. All calculations were carried out using Gaussian 09 computational program package. ${ }^{45}$

\section{Results and discussion}

\subsection{Regioselectivity study based on activation energies}

As outlined in the introduction, when an asymmetric 1,3-dipole and dipolarophile come into the reaction channel, depending on the direction of attack to each other, two regioisomers corresponding to two different transition structures can be generated. Potential energy surfaces (PES) for all reactions under investigation is schematically sketched in figure 1.

Activation energies $\left(E_{\mathrm{a}}\right)$ corresponding to the two pathways $(1,4$ and 1,5$)$ presented on figure 1 clearly show that 1,5-pathway is kinetically favoured for all studied reactions; that is, in these reactions, 1,5regioisomer is the preferred regioisomer. According to the values of activation energies, it can be concluded that cycloadditions of Dph with D1 $(\mathrm{X}=\mathrm{NH})$ and with $\mathrm{D} 3(\mathrm{X}=\mathrm{S})$ should take place approximately in 


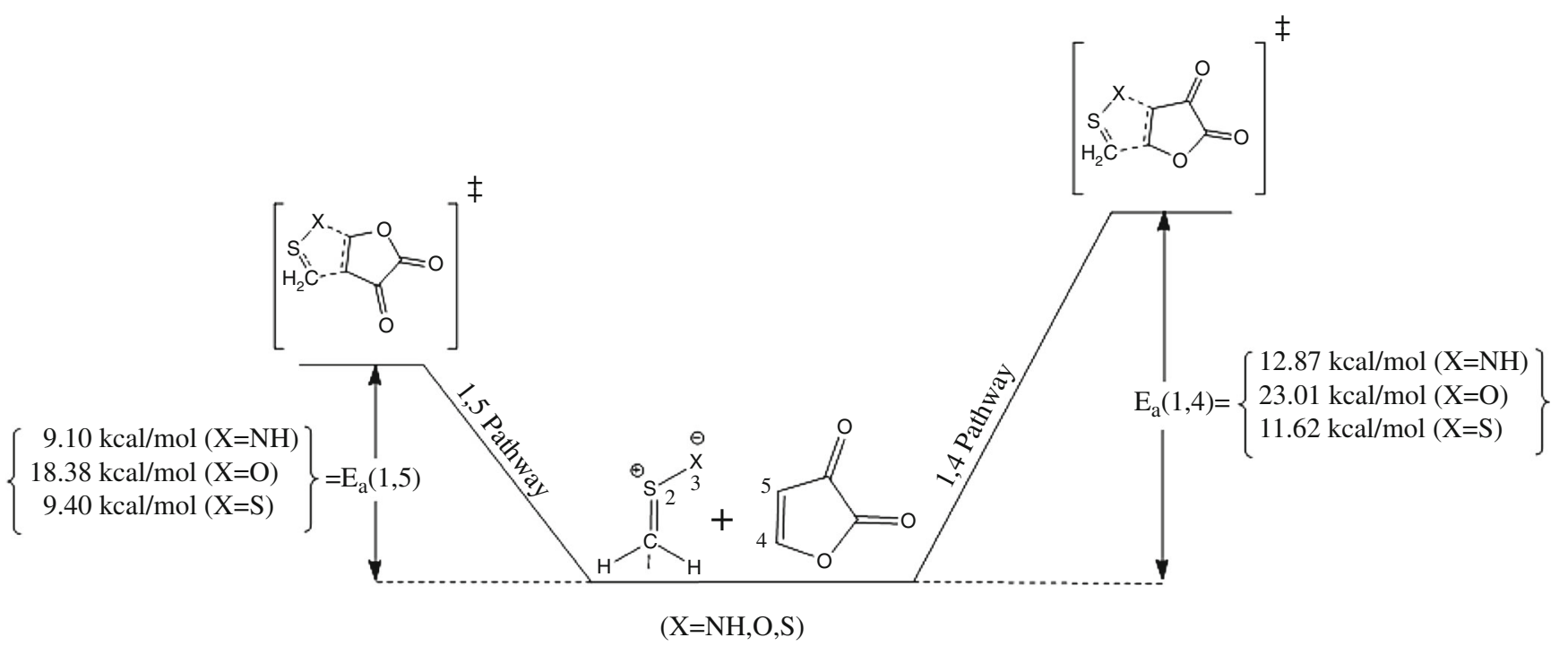

Figure 1. Schematic representation of potential energy surfaces (PES) corresponding to studied 1,3-DC reactions in two pathways.

the same rates while the reaction of Dph and D2 $(\mathrm{X}=\mathrm{O})$ should be the slowest one because of the largest activation energy. Furthermore, magnitude of the asynchronicity can be determined by considering the difference between the lengths of the two new $\sigma$ bonds which are being formed at the TSs; $|\Delta d|=\left|d_{1}-d_{2}\right|$. Values of $|\Delta d|$ in the TS $(1,4)$ and TS $(1,5)$ for cycloaddition of Dph with D1, D2 and with D3 are illustrated in figure 2. This figure demonstrates that both TSs are asynchronous and the order of asynchronicity in the predominant cycloaddition pathway, TS $(1,5)$, is: $\mathrm{D} 1(\mathrm{X}=\mathrm{NH})>\mathrm{D} 2(\mathrm{X}=\mathrm{O})>\mathrm{D} 3(\mathrm{X}=\mathrm{S})$.

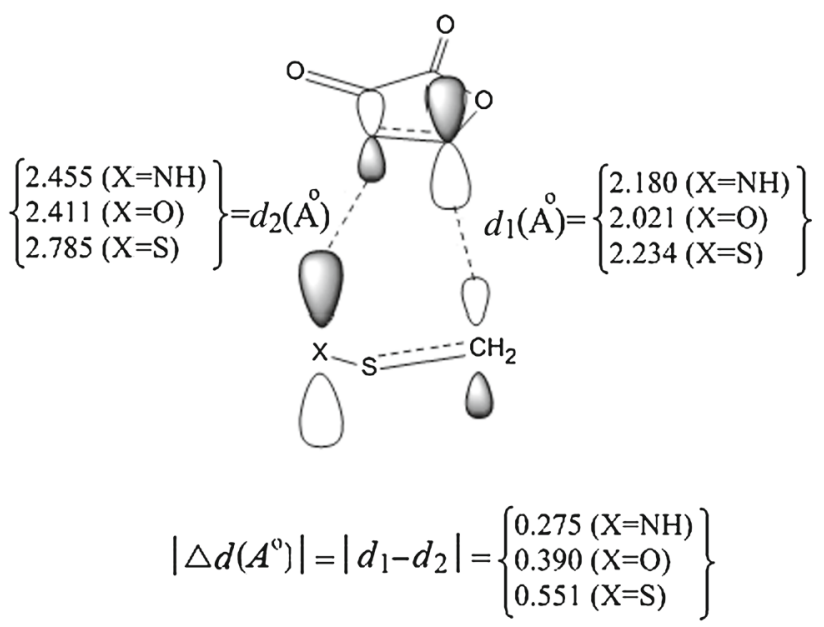

\subsection{Regioselectivity study based on DFT reactivity indices}

Prediction of regioselectivity is necessarily dependent on determination of NED/IED character of reaction. This characterization can be performed by using electronic chemical potential $(\mu)$, electrophilicity index $(\omega)$, charge transfer (CT) analysis in the TSs, and HOMO-LUMO gap between two reactants. An important point which must be considered in determination of HOMO-LUMO gaps in the 1,3-DC reactions is that HOMO and LUMO are the orbitals involved directly

Figure 2. Values of $|\Delta d|$ in TS $(1,4)$ and TS $(1,5)$ for the 1,3-DC reaction of Dph with $\mathrm{D} 1(\mathrm{X}=\mathrm{NH}), \mathrm{D} 2(\mathrm{X}=\mathrm{O})$ and $\mathrm{D} 3$ $(\mathrm{X}=\mathrm{S})$. 


\section{HOMO}
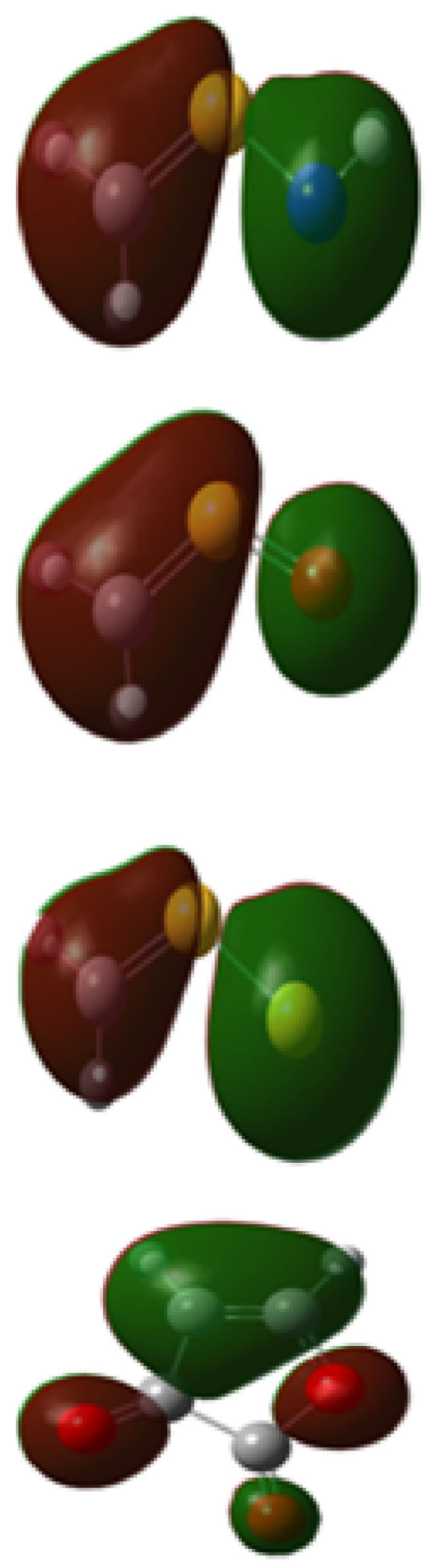

LUMO

D1

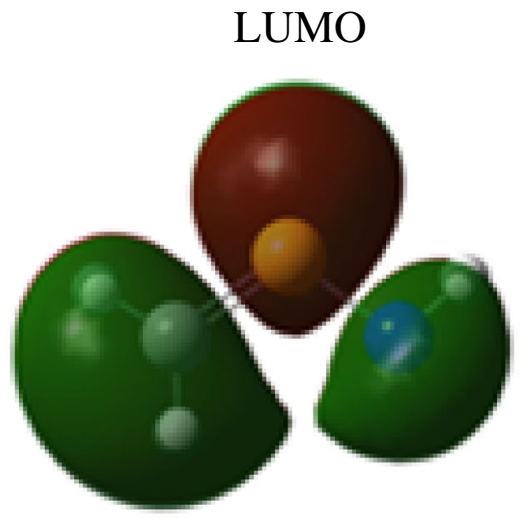

D2

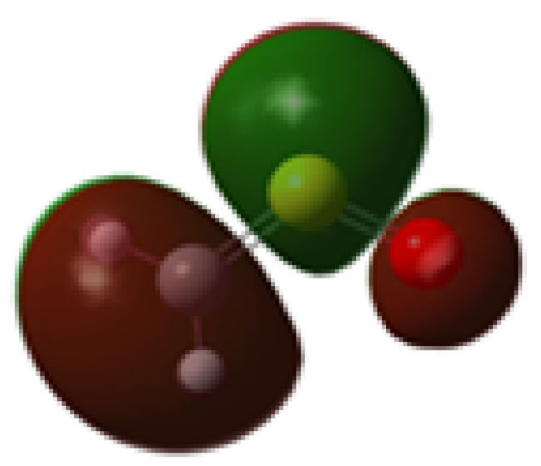

D3

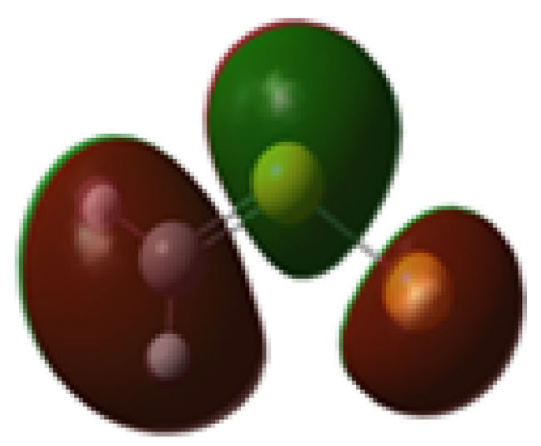

Dph

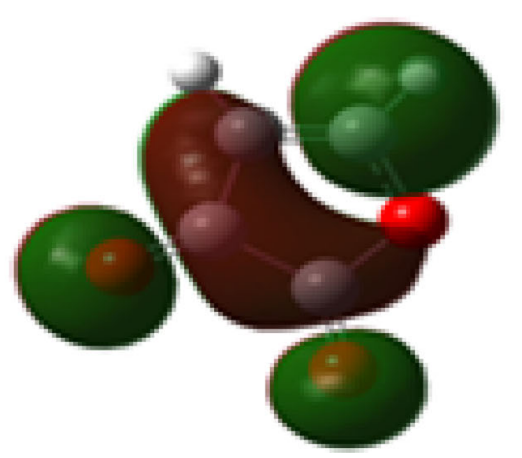

Figure 3. Optimized geometries and visualized FMOs for the reactants.

in the reaction (the relevant HOMO and LUMO) and not necessarily the highest occupied and lowest unoccupied molecular orbitals (the actual HOMO and
LUMO) so that the relevant HOMO and LUMO are the highest occupied $\pi$ molecular orbital and the lowest unoccupied $\pi$ molecular orbital $\left(\pi^{*}\right)$, respectively. 
Table 1. FMO energies (a.u.), electronic chemical potential (a.u.), chemical hardness (a.u.), chemical softness (a.u. ${ }^{-1}$ ) and electrophilicity index (a.u.) for the reactants calculated at the $\mathrm{HF} / 6-311++\mathrm{G}^{* *}$ //B3LYP/6-311++G** level.

\begin{tabular}{lcrrccc}
\hline Reactant & HOMO & \multicolumn{1}{c}{ LUMO } & $\mu$ & $\eta$ & $S$ & $\omega$ \\
\hline D1 & -0.32233 & 0.03904 & -0.1416 & 0.1806 & 2.7685 & 0.0555 \\
D2 & -0.38015 & 0.02779 & -0.1761 & 0.2039 & 2.4521 & 0.0760 \\
D3 & -0.32387 & -0.00293 & -0.1634 & 0.1604 & 3.1172 & 0.0832 \\
Dph & -0.40838 & 0.02009 & -0.1941 & 0.2142 & 2.3342 & 0.0879 \\
\hline
\end{tabular}

Figure 3 represents optimized geometries and visualized frontier molecular orbitals (HOMOs and LUMOs) of reactants. In the present study, based on the HF/6$311++\mathrm{G}^{* *}$ single point calculations on the B3LYP/6$311++\mathrm{G}^{* *}$ optimized geometries, there are no differences between the relevant and actual FMOs for the reactants. The electronic chemical potential (table 1) of Dph (-0.1941 a.u.) is more negative than that of 1,3dipoles D1-D3. Consequently, charge transfer will take place from the 1,3-dipoles to DPh and this is also in agreement with the charge transfer analysis in table 2 . A closer look at the values of electrophilicity indices $(\omega)$ in the last column of table 1 reveals that Dph, in comparison with D1-D3, acts as an electrophile due to the larger value of its $\omega$. Table 1 indicates the values of the FMO energies (a.u.) and the two possible interactions between the FMOs (LUMO) $)_{\mathrm{DPh}}-(\mathrm{HOMO})_{1,3-\text { dipole }}$ and $(\mathrm{LUMO})_{1,3-\text { dipole }}-(\mathrm{HOMO})_{\mathrm{DPh}}$ for the cycloadditions of Dph with D1-D3 are shown in figure 4.

As can be seen, for all cyclizations, the values of $\triangle E$ (HOMO-LUMO gap) corresponding to NED are lower than that of $\Delta E$ values corresponding to IED. Furthermore, an excellent linear correlation, $R^{2}=1.00$, between the values of $\Delta E$ corresponding to NED and activation energies in the predominant pathway can be observed. This excellent correlation is plotted in figure 5. Consequently, based on the aforementioned, Fukui functions for electrophilic $\left(f_{k}^{-}\right)$and nucleophilic $\left(f_{k}^{+}\right)$attacks should be calculated for D1-D3 and Dph, respectively. It is well known that Fukui functions values obtained from various population schemes may provide negative values; whereas, Hirshfeld's population scheme guarantees positive Fukui functions values. $^{46,47}$ Therefore, Fukui functions based on the Hirshfeld population for reactants were calculated and corresponding results are tabulated in table 3. Regioselectivity in the 1,3-DC reactions can be explained using the HSAB principle, which is also recognized as Gazquez-Mendez rule: ${ }^{48}$ the interaction between two chemical species is more favoured when the softness difference of two interacting atoms is minimum. ${ }^{49}$ Values of $\Delta S$ collected in table 4 for both pathways were computed using the following formulas (see figure 1 for atom numbering):

$$
\begin{aligned}
& \Delta S(1,4)=\left(s_{\mathrm{X} 3}^{-}-s_{\mathrm{C} 5}^{+}\right)^{2}+\left(s_{\mathrm{C} 1}^{-}-s_{\mathrm{C} 4}^{+}\right)^{2} \\
& \Delta S(1,5)=\left(s_{\mathrm{X} 3}^{-}-s_{\mathrm{C} 4}^{+}\right)^{2}+\left(s_{\mathrm{C} 1}^{-}-s_{\mathrm{C} 5}^{+}\right)^{2} .
\end{aligned}
$$

As the values of $\Delta S$ reveal, the reaction channel with lower activation energy corresponding to predominant regioisomer is associated with a smaller $\Delta S$ value. Hence, the success of Gazquez-Mendez rule based on

Table 2. Charge transfer, $\mathrm{CT}^{\mathrm{a}}$ in e, from 1,3-dipole to dipolarophile in the

\begin{tabular}{|c|c|c|c|c|c|c|}
\hline \multirow[b]{2}{*}{ Reaction } & \multicolumn{2}{|c|}{$\mathrm{NPA}^{\mathrm{b}}$} & \multicolumn{2}{|c|}{$\mathrm{MK}^{\mathrm{c}}$} & \multicolumn{2}{|c|}{ Hirshfeld } \\
\hline & $\mathrm{TS}(1,4)$ & $\mathrm{TS}(1,5)$ & $\mathrm{TS}(1,4)$ & $\mathrm{TS}(1,5)$ & $\mathrm{TS}(1,4)$ & $\mathrm{TS}(1,5)$ \\
\hline $\mathrm{D} 1+\mathrm{Dph}$ & 0.1004 & 0.1651 & 0.1082 & 0.1735 & 0.1142 & 0.2059 \\
\hline $\mathrm{D} 2+\mathrm{Dph}$ & 0.0303 & 0.0120 & 0.0313 & 0.0247 & 0.0310 & 0.0407 \\
\hline D3+Dph & 0.0940 & 0.1119 & 0.0752 & 0.0646 & 0.0409 & 0.0453 \\
\hline
\end{tabular}
studied reactions.

a: residual charge on the 1,3-dipole moiety has been considered b: natural population analysis c: Merz-Kollman 


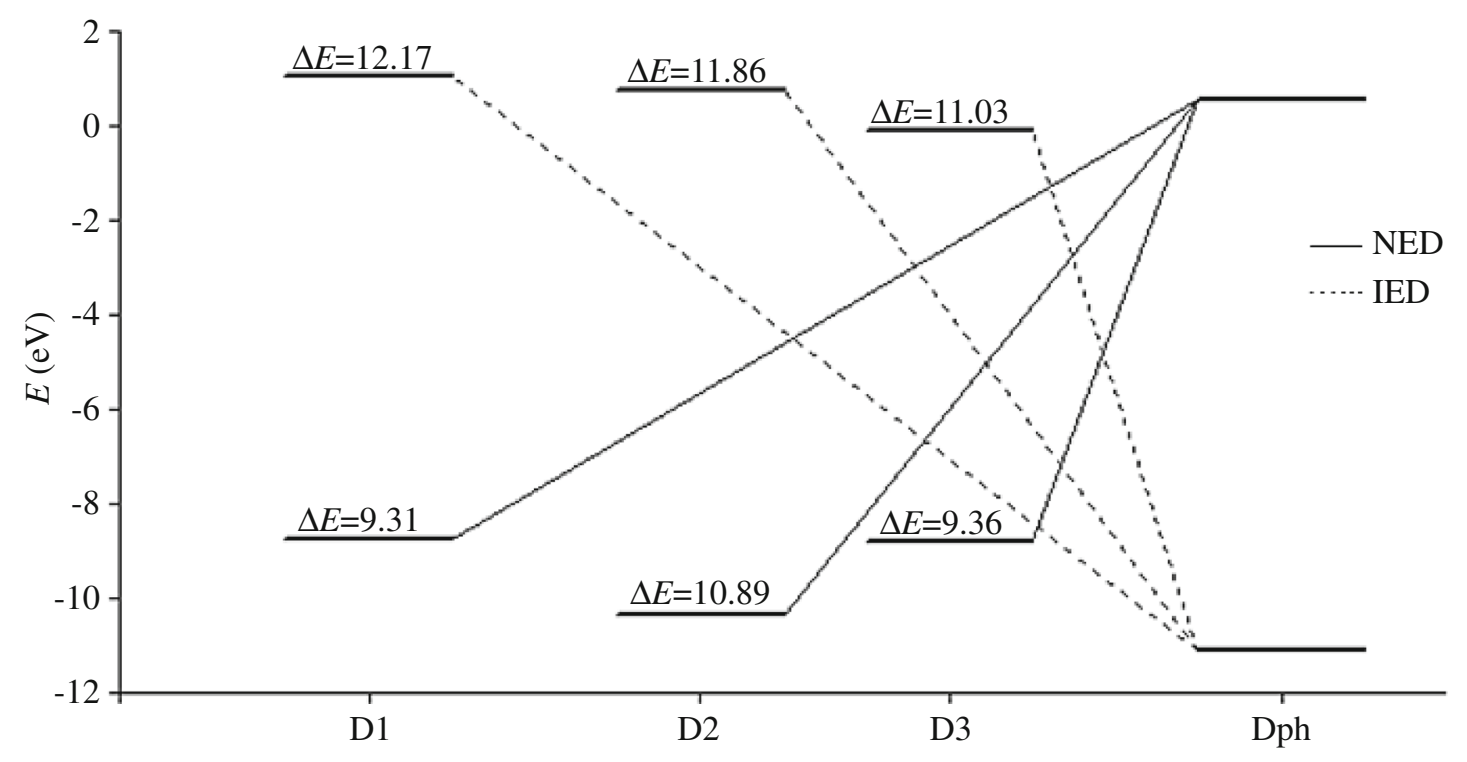

Figure 4. FMO interactions in the 1,3-DC reaction of Dph with D1, D2 and D3.

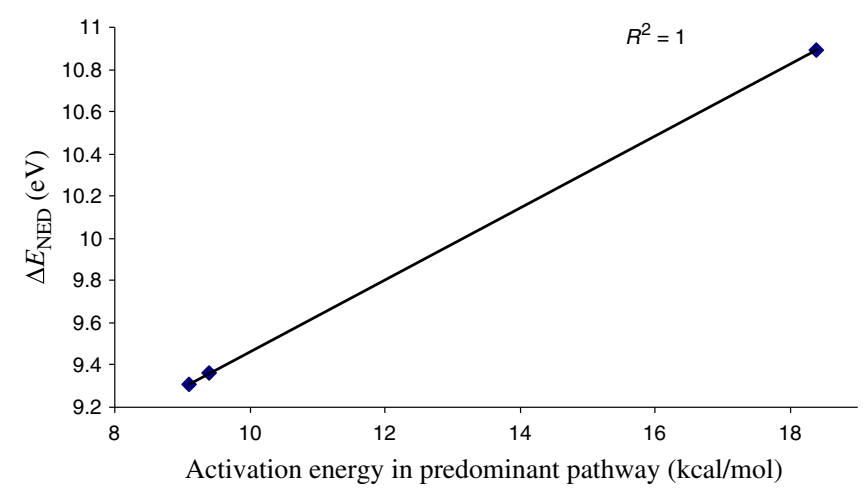

Table 4. Values of $\Delta S(1,4)$ and $\Delta S(1,5)$ for the studied reactions calculated based on Hirshfeld's population.

\begin{tabular}{lcc}
\hline Reaction & $\Delta S(1,4)$ & $\Delta S(1,5)$ \\
\hline D1+Dph & 0.429 & 0.404 \\
D2+Dph & 0.396 & 0.349 \\
D3+Dph & 2.369 & 1.959 \\
\hline
\end{tabular}

Figure 5. Linear correlation between $\Delta E$ values corresponding to NED mechanism and calculated activation energies in a predominant pathway.

Table 3. Local descriptors (Fukui function, local softness and local philicity power) in the reaction sites of the reactants calculated based on the Hirshfeld's population (see figure 1 for atom numbering).

\begin{tabular}{|c|c|c|c|c|c|c|c|c|}
\hline \multirow{2}{*}{$\begin{array}{l}\text { Local } \\
\text { descriptor }\end{array}$} & \multicolumn{2}{|c|}{ D1 } & \multicolumn{2}{|c|}{ D2 } & \multicolumn{2}{|c|}{ D3 } & \multicolumn{2}{|c|}{ Dph } \\
\hline & $\mathrm{C}_{1}$ & $\mathrm{X}_{3}$ & $\mathrm{C}_{1}$ & $\mathrm{X}_{3}$ & $\mathrm{C}_{1}$ & $\mathrm{X}_{3}$ & $\mathrm{C}_{4}$ & $\mathrm{C}_{5}$ \\
\hline$f^{+}$ & - & - & - & - & - & - & 0.143 & 0.074 \\
\hline$f^{-}$ & 0.239 & 0.267 & 0.244 & 0.303 & 0.139 & 0.548 & - & - \\
\hline$s^{+}$ & - & - & - & - & - & - & 0.333 & 0.172 \\
\hline$s^{-}$ & 0.661 & 0.739 & 0.598 & 0.743 & 0.433 & 1.708 & - & - \\
\hline$\omega^{+}$ & - & - & - & - & - & - & 0.012 & 0.006 \\
\hline$\omega^{-}$ & 0.013 & 0.014 & 0.018 & 0.023 & 0.011 & 0.045 & - & - \\
\hline
\end{tabular}




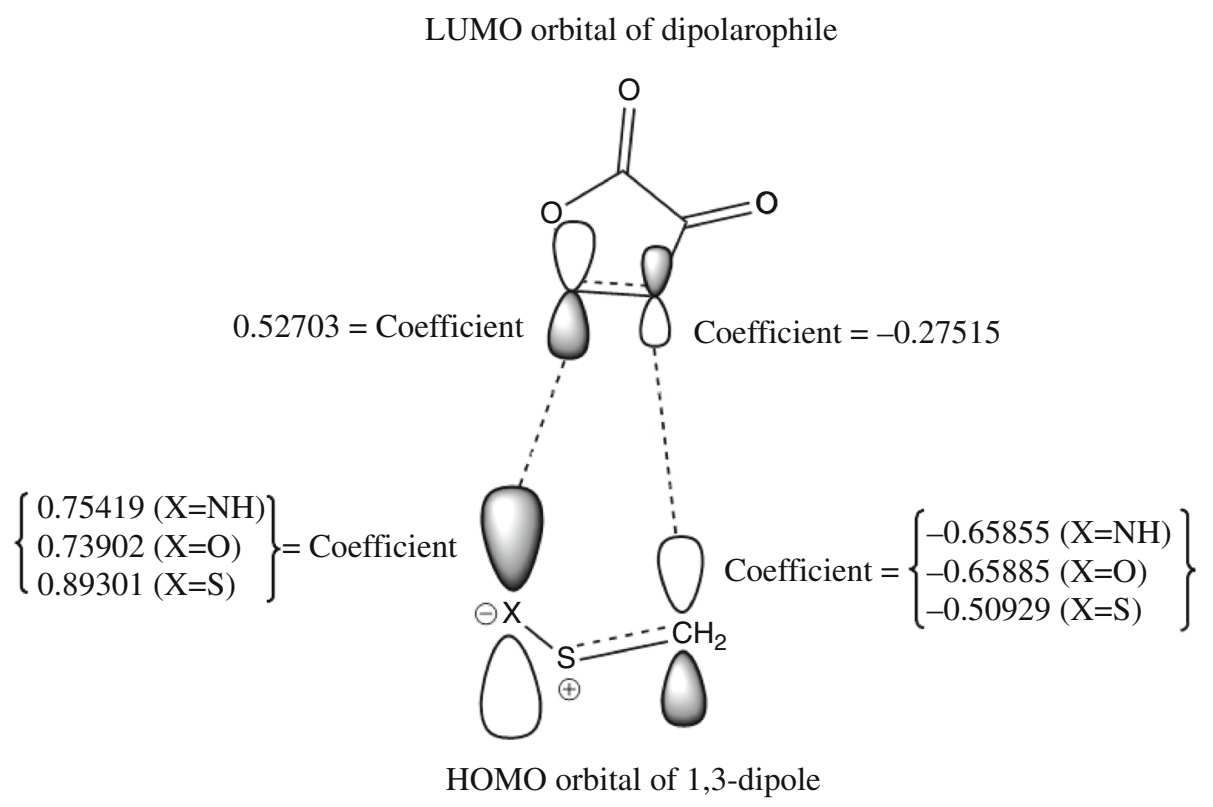

Figure 6. Coefficients of FMOs and illustration of Houk's rule.

the Hirshfeld analysis to predicting the true regioselectivity in the reactions under study is clarified. Prediction of regioselectivity can also be performed by using the Chattaraj's polar model. ${ }^{34}$ In this model, local philicity indices are used for prediction of regioselectivity in the 1,3-DC reactions. Values of local electrophilicity power, $\omega^{+}$, for atoms $\mathrm{C}_{4}$ and $\mathrm{C}_{5}$ of $\mathrm{DPh}$ and local nucleophilicity power, $\omega^{-}$, for atoms $\mathrm{C}_{1}$ and $\mathrm{X}_{3}$ of D1-D3 are presented in table 3 . In cycloaddition of Dph and D1-D3, the most favoured interactions should be considered between $\mathrm{C}_{4}$ of $\mathrm{Dph}$ (possessing the highest value of $\omega^{+}$) and $X_{3}$ of D1-D3 (possessing the highest value of $\omega^{-}$) which is, again, in accordance to generating the 1,5-regioisomer as a preferred one.

\subsection{Regioselectivity study based on Houk's rule}

According to the Houk's rule, regioselectivity of 1,3DC reactions can be explained on the basis that "the large-large and small-small FMO interactions are more favoured than that of large-small and small-large FMO interactions". FMO coefficients of frontier molecular orbitals of the reactants involved in the investigated reactions are given in figure 6 and clearly show that the cyclizations lead to the formation of 1,5-regioisomers as the major product in the studied 1,3-DC reactions.

\subsection{Regioselectivity study based on MHP}

Values of hardnesses for the transition state structures and activation hardness corresponding to two regioisomeric pathways were calculated and tabulated in table 5. As can be observed, in all studied 1,3-DC reactions, TS $(1,5)$ is harder than that of TS $(1,4)$ and hence easier to form, which is reflected in the smaller activation hardness value associated with TS $(1,5)$. Again, here also, the kinetically favoured 1,5-regioisomer is

Table 5. FMO energies (a.u.) and chemical hardness (a.u.) for the TS(1,4) and TS $(1,5)$ calculated at the $\mathrm{HF} / 6-311++\mathrm{G}^{* *} / / \mathrm{B} 3 \mathrm{LYP} / 6-311++\mathrm{G}^{* *}$ level.

\begin{tabular}{|c|c|c|c|c|c|c|c|c|}
\hline \multirow[b]{2}{*}{ Reaction } & \multicolumn{2}{|c|}{ HOMO } & \multicolumn{2}{|c|}{ LUMO } & \multicolumn{2}{|c|}{$\eta$} & \multicolumn{2}{|c|}{$\Delta \eta^{\neq}$} \\
\hline & $\mathrm{TS}(1,4)$ & $\operatorname{TS}(1,5)$ & $\mathrm{TS}(1,4)$ & $\mathrm{TS}(1,5)$ & $\operatorname{TS}(1,4)$ & $\operatorname{TS}(1,5)$ & $\mathrm{TS}(1,4)$ & $\mathrm{TS}(1,5)$ \\
\hline $\mathrm{D} 1+\mathrm{Dph}$ & -0.33382 & -0.33428 & -0.01175 & -0.00766 & 0.1610 & 0.1633 & 0.2338 & 0.2315 \\
\hline $\mathrm{D} 2+\mathrm{Dph}$ & -0.34149 & -0.35635 & -0.04583 & -0.00103 & 0.1478 & 0.1776 & 0.2703 & 0.2405 \\
\hline D3+Dph & -0.34413 & -0.34731 & -0.04047 & -0.01190 & 0.1518 & 0.1677 & 0.2228 & 0.2069 \\
\hline
\end{tabular}


corresponded to the harder TS $(1,5)$ which is also in agreement with the MHP.

\section{Conclusion}

According to the computations carried out in this study, the following points can be concluded:

(i) Based on the calculated activation energies all studied reactions, especially in the predominant pathway, can take place with acceptable rates at room temperature; so that, the cycloaddition reactions of Dph with D1 $\left(E_{\mathrm{a}}=9.10 \mathrm{kcal} / \mathrm{mol}\right)$ and with D3 $\left(E_{\mathrm{a}}=9.40 \mathrm{kcal} / \mathrm{mol}\right)$ occur approximately at the same rates, whereas this cycloaddition with D2 $\left(E_{\mathrm{a}}=18.38 \mathrm{kcal} / \mathrm{mol}\right)$ should be the slowest one.

(ii) In the 1,3-DC reactions of Dph with D1-D3, in agreement with the concerted mechanism, the observed trend for the degree of asynchronicity in the predominant 1,5-pathway is: $\mathrm{D} 1(\mathrm{X}=\mathrm{NH})>$ $\mathrm{D} 2(\mathrm{X}=\mathrm{O})>\mathrm{D} 3(\mathrm{X}=\mathrm{S})$.

(iii) DFT global reactivity indices, charge transfer analysis in the TSs, and FMO interactions reveal that all cyclizations under investigation have NED character.

(iv) An excellent linear correlation, $R^{2}=1.00$, was obtained between FMO interactions ( $\Delta E$ values corresponding to NED) and calculated activation energies for studied reactions. On the other hand, activation energies in the predominant pathway are in the order of: D2 $\left(E_{\mathrm{a}}=\right.$ $18.38 \mathrm{kcal} / \mathrm{mol})>\mathrm{D} 3\left(E_{\mathrm{a}}=9.40 \mathrm{kcal} / \mathrm{mol}\right)>\mathrm{D} 1$ $\left(E_{\mathrm{a}}=9.10 \mathrm{kcal} / \mathrm{mol}\right)$, which are in harmony with the trend of: $\mathrm{D} 2(\Delta E=10.89 \mathrm{eV})>\mathrm{D} 3(\Delta E=$ $9.36 \mathrm{eV})>\mathrm{D} 1(\Delta E=9.31 \mathrm{eV})$.

(v) MHP, Chattaraj's polar model, Houk's rule and the Gazquez-Mendez rule confirm the resultant regioselectivity based on the calculated activation energies; that is, an excellent harmony can be observed between kinetic results and the electronic approaches.

\section{References}

1. Muchall R C H M and Peslherbe G H 2005 Can. J. Chem. 831615

2. Emamian S R and Zahedi E 2012 J. Phys. Org. Chem. 25748

3. Tanaka J and Kanemasa S 2001 Tetrahedron 57899

4. Huisgen R, Grashey R and Sauer J 1964 Chemistry of alkenes (New York: Interscience)

5. Pérez P, Domingo L R, Aurell M J and Contreras R 2003 Tetrahedron $\mathbf{5 9} 3117$
6. Benchouk W and Mekelleche S M 2008 J. Mol. Struct. (THEOCHEM) $\mathbf{8 5 2} 46$

7. Huisgen R 1984 In 1,3-Dipolar cycloaddition chemistry, A Padwa (ed.) (New York: Wiley-Interscience)

8. Di Valentin C, Freccero M, Gandolfi R and Rastelli A 2000 J. Org. Chem. 656112

9. Huisgen R 1968 J. Org. Chem. 332291

10. Huisgen R 1976 J. Org. Chem. 41403

11. Carey F A and Sundberg R J 2007 Advanced organic chemistry, 5th edn, (New York: Springer)

12. Fleming I 1976 Frontier orbitals and organic chemical reactions (London: Wiley)

13. Sustmann R and Shubert R 1972 Tetrahedron Lett. 13 4271

14. Sustmann R 1974 Pure Appl. Chem. 40569

15. Domingo L R, Aurell M J, Arnó M A and Sáez J A 2007 J. Mol. Struct. (THEOCHEM) $\mathbf{8 1 1} 125$

16. Chandra A K and Nguyen M T 1998 J. Phys. Chem. A102 6181

17. Sharma P, Kumar A and Sahu V 2010 J. Phys. Chem. A114 1032

18. Parthasarathi R and Chattaraj P K 2004 Bioorgan. Med. Chem. 125533

19. Chattaraj P K and Sarkar U 1968 J. Am. Chem. Soc. 108 5708

20. Padmanabhan J, Parthasarathi R and Chattaraj P K 2006 Chem. Res. Toxicol. 19356

21. Xia Y, Yin D, Rong C, Xu Q, Yin D and Liu S 2008 J. Phys. Chem. A112 9970

22. Geerlings P, De Proft F and Langenaeker W 2003 Chem. Rev. 1031793

23. Pearson R G 1968 J. Chem. Educ. 45581

24. Noorizadeh S $2005 \mathrm{~J}$. Mol. Struct. (THEOCHEM) 71327

25. Zhou Z and Parr R G 1990 J. Am. Chem. Soc. 112 5720

26. Nguyen L T, De Proft F, Nguyen M T and Geerlings P 1999 J. Chem. Soc., Perkin Trans. 261249

27. Chemouri H and Mekelleche S M 2006 J. Theor. Comput. Chem. 5197

28. Chemouri H, Benchouk W and Mekelleche S M 2006 J. Theor. Comput. Chem. 5707

29. Padwa A and Pearson W H 2002 The chemistry of heterocyclic compounds, Volume 59: Synthetic applications of 1,3-dipolar cycloaddition chemistry toward heterocycles and natural products (New York: John Wiley and Sons, Inc.)

30. Houk K N 1975 Acc. Chem. Res. 8361

31. Chattaraj P K, Chamorro E and Fuentealba P 1999 Chem. Phys. Lett. 314114

32. Chattaraj P K, Sarker U and Ranjan Roy D 2006 Chem. Rev. 1062065

33. Koopmans T A 1934 Physica 1 1-6 104

34. Parr R G, Von Szentpaly L and Liu S 1999 J. Am. Chem. Soc. 1211922

35. Ghomri A and Mekelleche S M 2010 J. Mol. Struct. (THEOCHEM) 94136

36. Yang W and Mortier W J 1986 J. Am. Chem. Soc. 108 5708

37. Chemouri H and Mekelleche S M 2007 J. Mol. Struct. (THEOCHEM) $\mathbf{8 1 3} 67$

38. Aurell M J, Domingo L R, Pérez P and Contreras R 2004 Tetrahedron 6011503 
39. Lee C, Yang W and Parr R G 1988 Phys. Rev. B37 785

40. Gordon M H and Pople J A 1988 J. Chem. Phys. 89 5777

41. Ess D H and Houk K N 2008 J. Am. Chem. Soc. 130 10187

42. Reed A E and Weinhold F 1983 J. Chem. Phys. 784066

43. Hirshfeld F L 1977 Theor. Chim. Acta. 44129

44. Besler B H, Merz K M J and Kollman P A 1990 J. Comp. Chem. 11431
45. Frisch M J et al. Gaussian 09 Revision-A.02 SMP, Gaussian Inc., Wallingford CT, 2009

46. Padmanabhan J, Parthasarathi R, Sarkar U, Subramanian V and Chattaraj P K 2004 Chem. Phys. Lett. 383122

47. Roy R K, Pal S and Hirao K 1999 J. Chem. Phys. 110 8236

48. Gazquez J L and Mendez F 1994 J. Phys. Chem. 984591

49. Chandra A K and Nguyen M 2002 Int. J. Mol. Sci. 3310 